\title{
Band gap modulation in zirconium-based metal-organic frameworks by defect engineering
}

Received 00th January 20xx, Accepted 00th January 20xx DOI: $10.1039 / \times 0 \times x 00000 x$

\author{
Marco Taddei, ${ }^{*, a}$ Giulia M. Schukraft, ${ }^{\text {b }}$ Michael E. A. Warwick, ${ }^{a}$ Davide Tiana,, ${ }^{*, c}$ Matthew J. \\ McPherson, ${ }^{a}$ Daniel R. Jones ${ }^{a}$ and Camille Petit ${ }^{b}$
}

\begin{abstract}
We report a defect-engineering approach to modulate the band gap of zirconium-based metal-organic framework UiO-66, enabled by grafting of a range of amino-functionalised benzoic acids at defective sites. Defect engineered MOFs were obtained by both post-synthetic exchange and modulated synthesis, featuring band gap in the 4.1-3.3 eV range. First principle calculations suggest that shrinking of the band gap is likely due to an upward shift of the valence band energy, as a result of the presence of lightabsorbing monocarboxylates. The photocatalytic properties of defect-engineered MOFs towards $\mathrm{CO}_{2}$ reduction to $\mathrm{CO}$ in the gas phase and degradation of Rhodamine $B$ in water were tested, observing improved activity in both cases, in comparison to a defective UiO-66 bearing formic acid as the defect-compensating species.
\end{abstract}

Defect engineering has recently emerged as a new trend for tuning the physical-chemical properties of metal-organic frameworks (MOFs). ${ }^{1-3}$ Among other families within the MOF realm, zirconium-based MOFs (Zr-MOFs) have gained the spotlight as the most attractive platform for defect engineering, owing to their ability to retain their stability even in the presence of large quantities of defects. ${ }^{4}$ This peculiar characteristic of $\mathrm{Zr}$-MOFs is attributed to the high degree of connectivity between the hexanuclear $\left[\mathrm{Zr}_{6} \mathrm{O}_{4}(\mathrm{OH})_{4}\right]^{12+}$ clusters, which allows the framework to tolerate the removal of part of the linkages without undergoing collapse. This has inspired the rapid development of synthetic approaches to introduce defects in a controlled manner and to exploit these defects as an alternative pathway to impart new functions to the framework. ${ }^{5-9}$ The most straightforward method to generate defects within $\mathrm{Zr}$-MOFs is coordination modulation, i.e. the addition to the reaction mixture of superstoichiometric

\footnotetext{
a. Energy Safety Research Institute, Swansea University, Fabian Way, Swansea, SA1 8EN, UK.E-mail: marco.taddei@swansea.ac.uk

b. Barrer Centre, Department of Chemical Engineering, Imperial College London, South Kensington Campus, SW7 2AZ London, UK.

c. School of Chemistry, University College Cork, College Road, Cork T12 K8AF, Ireland.Email: davide.tiana@ucc.ie

Electronic Supplementary Information (ESI) available: [Details on the following: synthesis and characterisation; computational details; photocatalytic $\mathrm{CO}_{2}$ reduction; photocatalytic dye degradation]. See DOI: 10.1039/x0xx00000x
}

amounts of monocarboxylic acids (typically formic acid, acetic acid, benzoic acid) that compete with polycarboxylic linkers for coordination to the metal clusters. ${ }^{10}$ Modulators slow down crystallisation kinetics and, more importantly, can be retained within the structure in place of the linkers, thus creating defects, most often of missing-cluster nature. ${ }^{5}, 11$ The defectcompensating monocarboxylates can be subsequently exchanged post synthesis by soaking the defective MOF in a solution of the desired functional mono- or polycarboxylate, a process known as post-synthetic defect exchange (PSDE) (Figure S1). ${ }^{12}, 13$ The resulting MOF is selectively modified at defect sites, with the functional backbone of the newly installed carboxylate exposed within the large cavity associated with a missing-cluster vacancy. Functionalisation of the defective MOF can alternatively be accomplished in one step, by employing the desired functional monocarboxylate as a modulator (Figure S2).9, 14, 15 This method is of practical interest, since it involves one less synthetic step than PSDE, but some functional monocarboxylates may not be suitable to act as coordination modulators, e.g. failing to provide a crystalline product or the desired crystalline phase. Irrespective of the method chosen to introduce functionalities in the MOF structure, the main advantage of defect engineering is that this approach potentially provides access to a much larger chemical space than the classical method based on the use of modified linkers. This is mainly because any species containing at least one carboxylic acid group, and having size not exceeding the size of the cavity generated by a missing-cluster defect, could in principle be employed to functionalise the defective MOF. On the other hand, a functionalised linker must have well-defined features, in terms of length and geometry, to be incorporated in a non-defective framework, which poses restrictions to the range of accessible functional groups.

We have recently explored the potential of PSDE as a method to improve the $\mathrm{CO}_{2}$ capture performance of defective UiO$66,{ }^{13}$ the prototypical Zr-MOF based on 1,4benzenedicarboxylic acid (or terephthalic acid, hereafter BDC) as the linker. In the present work, we focus instead on providing proof of concept that the band gap of UiO-66 can be 
systematically modulated through defect engineering. Several reports in the literature ${ }^{16-20}$ have shown that replacement of $B D C$ linker with aminoterephthalate (ABDC) in UiO-66 reduces the band gap from about $4.0 \mathrm{eV}$ to about 2.8-2.9 eV, mainly thanks to the generation of a new highest occupied crystalline orbital (HOCO), predominantly localised on the organic linker. Inspired by these reports, we set out to investigate whether a similar result could be achieved by grafting of a range of commercially available and inexpensive amino-functionalised benzoic acids (ABAs, Figure 1) at defective sites, and how the relative arrangement of the carboxylic and amine groups within the phenyl ring would affect the electronic structure of the framework. The selected ABAs are 2-aminobenzoic acid (2ABA), 3-aminobenzoic acid (3ABA), 4-aminobenzoic acid (4ABA) and 3,5-diaminobenzoic acid (35DABA). Benzoic acid (BA) was also employed, in order to assess the effect of grafting a non-functionalised aromatic ring at defective sites on the properties of the framework.

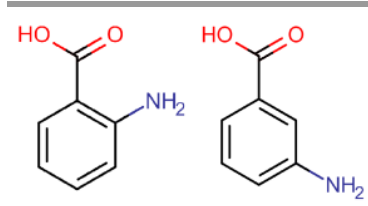

2ABA

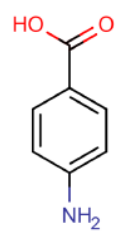

$4 \mathrm{ABA}$

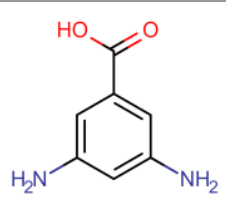

35DABA

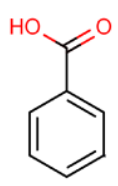

BA
Figure 1. Structural formulas of 2-aminobenzoic acid (2ABA), 3-aminobenzoic acid (3ABA), 4-aminobenzoic acid (4ABA), 3,5-diaminobenzoic acid (35DABA) and benzoic acid (BA).

In order to have a range of model systems that could allow isolating the effect of a single variable, i.e. the position and number of amine groups of the defect-compensating species, we initially prepared defect-engineered MOFs by PSDE, using the same defective MOF as a matrix for exchange. We prepared a batch of defective, formic acid modulated UiO-66, named FA_mod (see ESI for details on synthesis and characterisation, Figures S3-8, Tables S1-3). FA_mod was then soaked in $\mathrm{N}, \mathrm{N}$-dimethylformamide (DMF) solutions of the ABAs displayed in Figure 1. Herein, we use the nomenclature FA_mod- $X$ to refer to samples prepared through PSDE, starting from FA_mod, where $X$ can be either BA, 2ABA, 3ABA, 4ABA or 35DABA. PSDE led to a substantial decrease in surface area and pore volume of the MOF and to the disappearance of the large pores having diameter of about $8.5 \AA$, which proves that the new functional monocarboxylates have successfully been installed at defective sites (Figures S5-6, Table S1). NMR analysis of the dissolved solids in $1 \mathrm{M} \mathrm{NaOH}$ in $\mathrm{D}_{2} \mathrm{O}$ further confirms that FA was replaced almost completely (Figures S913), while the crystallinity was fully retained, as demonstrated by powder X-ray diffraction (PXRD) patterns (Figure S3). TGA shows that introduction of $A B A s$ decreased the thermal stability of the framework in the order FA_mod = FA_mod-BA $>$ FA_mod-4ABA > FA_mod-3ABA > FA_mod-2ABA > FA_mod35DABA (Figure S6). We also attempted to introduce 2,3diaminobenzoic acid (23DABA), 3,5-diaminobenzoic acid (25DABA) and 3,4-diaminobenzoic acid (34DABA) by PSDE in the same conditions used for the other $A B A s$. The resulting solids were strongly coloured after exchange (dark red, dark purple and beige, respectively) and fully retained crystallinity (Figure S14). However, NMR of the digested MOFs showed little or no presence of the desired $A B A$, even though the $\mathrm{BDC} / \mathrm{FA}$ ratio considerably decreased in all cases (Figures S1517).

To investigate the optoelectronic properties of the materials, diffuse reflectance UV-Visible spectroscopy was carried out (Figure 2). A clear red shift of the absorption frequency onset is observed, in the order FA_mod = FA_mod-BA < FA_mod4ABA < FA_mod-3ABA < FA_mod-2ABA < FA_mod-35DABA. Interestingly, this order mirrors what was observed for thermal stability, suggesting that reduction of band gap is accompanied by destabilisation of the framework (Figure S6). FA_mod35DABA shows a broad absorption feature throughout the visible region, which is reflected by the brown colour of the powder. The band gap for each compound was estimated from the relative Tauc plots (Figure S18), obtaining the values reported in Table 1 . These results prove that systematic modulation of the band gap can be achieved altering either the position of the amino groups or their number in the aromatic ring.

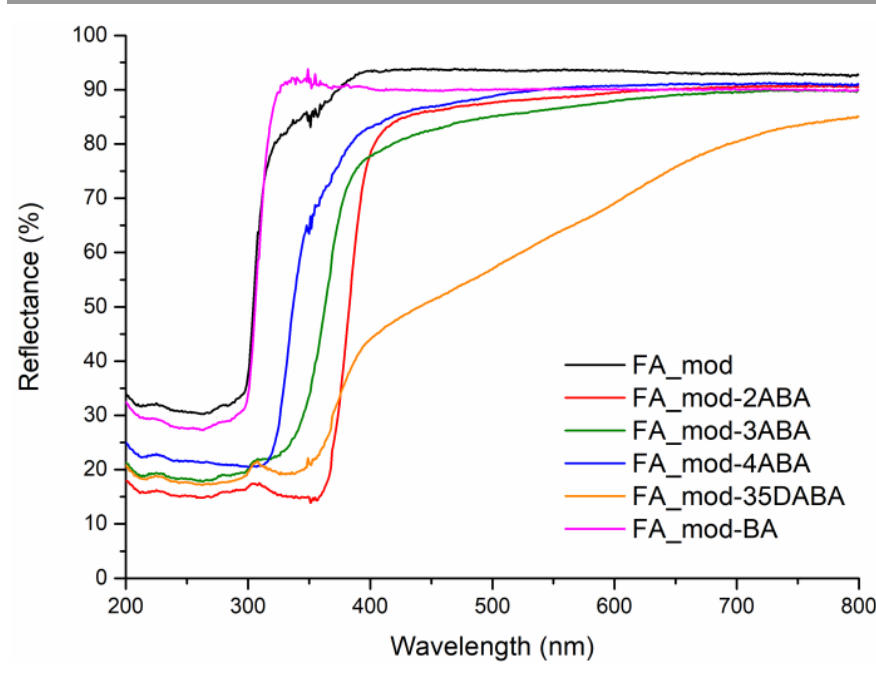

Figure 2. Diffuse reflectance UV-Vis spectra for FA_mod (black), FA_mod-2ABA (red) FA_mod-3ABA (olive), FA_mod-4ABA (blue), FA_mod-35DABA (orange) and FA_modBA (magenta).

Comparison of the reflectance spectra for each MOF and the corresponding free ABA (Figures S19-22) shows that their photochemical behaviour is very similar, suggesting that coordination of the acid to the metal clusters does not significantly affect their ability to interact with light. In order to test the stability of the defect-engineered MOFs under prolonged irradiation, the powders were kept for $18 \mathrm{~h}$ under combined visible and UV (365 nm) light. This treatment led to a colour change, especially evident for FA_mod-2ABA, FA_mod-3ABA and FA_mod-4ABA (Figure S23), and also evidenced by UV-Vis spectroscopy, displaying the appearance of a broad absorption feature in the visible region (Figures S2427). Thermal treatment at $150{ }^{\circ} \mathrm{C}$ for 4 hours did not produce the same effect, suggesting that it is purely induced by light. The process did not affect structural integrity of the MOFs (as 
demonstrated by PXRD, Figures S28-31), but it did lead to a change in composition, as suggested by the increase of BDC/ABA ratio observed by NMR analysis (Figures S32-35). Even though the powders were not washed before being dissolved for NMR analysis, we did not observe significant signals attributable to other species than $B D C$ or the $A B A$. FA_mod-23DABA, FA_mod-25DABA and FA_mod-34DABA all showed strong absorption in the visible (Figures S36-38) and low band gaps of 2.1, 1.7 and $2.4 \mathrm{eV}$, respectively (Figure S39). However, in the absence of clear evidence from NMR of the effective presence of the ABAs is these samples, we decided not to further characterise them. In order to have a term of comparison for our defect-engineered MOFs, we prepared a sample of mixed-linker UiO-66 incorporating 33\% of ABDC (UiO-66- $\mathrm{NH}_{2} 33 \%$ ), featuring the same $-\mathrm{NH}_{2} / \mathrm{Zr}$ ratio of FA_mod-2ABA (Figures S40-43). The band gap determined from the Tauc plot of UiO-66- $\mathrm{NH}_{2} 33 \%$ is $3.0 \mathrm{eV}$ (Figure S44), nearly the same as that usually reported for fully functionalised UiO-66- $\mathrm{NH}_{2}(2.8-2.9 \mathrm{eV}) .{ }^{18,} 20$

Table 1. Band gap values, as extracted from Tauc plots, and calculated HOMO-LUMO gaps for FA_mod and corresponding defect-engineered MOFs, presented in decreasing order. A similar trend (i.e. the gap shrinks) can be found in both experimental and calculated gaps. The calculations were done at PBE level, which underestimates the band-gap and explains the discrepancy in the absolute values. Higher level of theory could not be used due to the size of the system.

\begin{tabular}{ccc}
\hline Sample & $\begin{array}{c}\text { Experimental } \\
\text { band gap } \\
(\mathrm{eV})\end{array}$ & $\begin{array}{c}\text { Calculated } \\
\text { HOMO-LUMO gap } \\
(\mathrm{eV})\end{array}$ \\
\hline Defect-free UiO-66 & $4.1^{17}$ & 3.2 \\
FA_mod & 4.1 & 3.2 \\
FA_mod-4ABA & 3.8 & 2.2 \\
FA_mod-3ABA & 3.5 & 1.9 \\
FA_mod-2ABA & 3.3 & 1.8 \\
FA_mod-35DABA & 3.3 & 1.6 \\
\hline
\end{tabular}

We also explored the possibility of preparing defectengineered MOF samples by modulated synthesis. To the best of our knowledge, only $4 \mathrm{ABA}$ was previously employed as a modulator, for both UiO-66 $66^{14}$ and phase-pure NU-90121 synthesis. 15 equivalents of each $A B A$ were employed as modulators, except for 35DABA, whose amount had to be increased to 25 equivalents to produce a MOF with acceptable crystallinity. These samples were named according to the formula $X$ _mod, where $X$ can be either 2ABA, 3ABA, 4ABA or 35DABA. PXRD analysis shows that 2ABA_mod and 4ABA_mod feature narrower diffraction peaks, indicative of larger crystallite size, than 3ABA_mod and 35DABA_mod (Figure S45). NMR analysis shows that a much larger amount of functional groups could be introduced via modulated synthesis than by PSDE, suggesting that much more defective frameworks were obtained (Figures S46-49, Table S4). However, $\mathrm{N}_{2}$ sorption isotherms reveal low BET surface areas and feature evident hysteresis, indicative of the presence of mesopores, for two MOFs out of four (Figure S50, Table S5). PXRD patterns after $\mathrm{N}_{2}$ sorption analysis show that all the samples suffered from loss of crystallinity (Figure S51). TGA shows that all the materials undergo decomposition of the framework at lower temperature than FA_mod, but they do not display major weight losses below $300{ }^{\circ} \mathrm{C}$ (Figure S52). Loss of crystallinity and porosity during activation could therefore be due to partial collapse of the highly defective frameworks upon removal of solvent when heated to $120{ }^{\circ} \mathrm{C}$ under vacuum. Despite the higher loading of photoactive groups, diffuse reflectance UV-Vis spectra for these samples do not largely differ from those of the samples obtained by PSDE (Figures S19-22).

To gain insight into the effect of defect engineering on the electronic structure of the framework, calculations were performed using the Time Dependent Density Functional level of Theory, which allows to study the electron excitation to the unoccupied orbitals (see ESI for description of the computational details). The defective UiO-66 frameworks were simulated using the cluster approximation, as previously done by some of us and other authors. ${ }^{17}, 22-25$ Specifically, we constructed fragments constituted of a hexanuclear cluster coordinated by eight terephthalates and four monocarboxylates, representative of the clusters found in the reo defective topology associated with missing-cluster defects. ${ }^{11}$ Table 1 reports the calculated gap between the highest occupied molecular orbital (HOMO) and the lowest unoccupied molecular orbital (LUMO), which can be reasonably approximated to the gap between the valence and the conduction band, respectively, in a solid. ${ }^{18}$ It is to be noted that, due to the size of the simulated clusters (ca 220 atoms and ca 850 electrons), the calculations could only be run using the generalized gradient approximations (GGA) functional Perdew, Burke and Ernzerhof (PBE), which is known to underestimate the band gap of UiO- $66 .{ }^{26}$ However, it can be used to rationalise and predict band gap, as well as to study frontier orbitals in MOFs. ${ }^{27}$ The results in table 1 indicate that there is no difference between a defect-free UiO-66 and a defective UiO-66 where defects are compensated by FA, i.e. FA_mod. Inclusion of ABAs as defect-compensating species shrinks the HOMO-LUMO gap. A trend depending on the position and on the number of $\mathrm{NH}_{2}$ can be noted, which is consistent with that experimentally observed by UV-Vis spectroscopy. For the pristine, defect-free, UiO-66 crystal the Highest Occupied Orbital (corresponding to the valence band) is a localised orbital composed of the oxygen $\mathrm{p}$ orbitals, ${ }^{19}$ while the Lowest Unoccupied Orbital (corresponding to the conduction band) is a fully delocalised orbital between the inorganic node and the organic ligand. ${ }^{18} \mathrm{~A}$ similar situation can be found in the HOMO and LUMO of the single-cluster model for defect free UiO-66 (Figure S53), which validates our computational model. Figure 3 shows the comparison of the frontier orbitals for a FA-compensated and a 2ABAcompensated defective model, representative of FA_mod and FA_mod-2ABA, respectively (see the ESI for more details). 

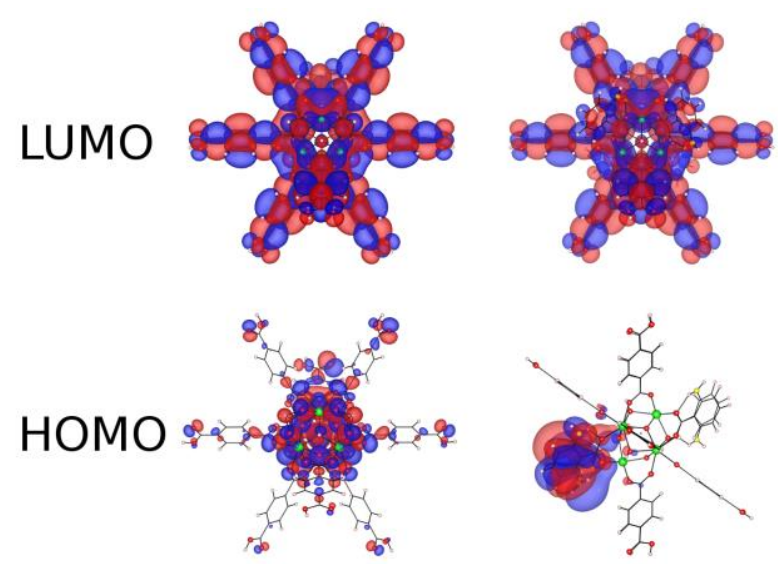

FA_mod

FA_mod-2ABA

Figure 3. Calculated HOMO (bottom) and LUMO (top) for FA_mod (left) and FA_mod2ABA (right).

A major change occurs in the HOMO, which becomes a set of two degenerate localised orbitals on the defect. On the other hand, the LUMO seems to be basically unaffected by the changes occurring at defective sites. A Similar situation is encountered when a range of mono- and disubstituted benzoic acids are present as defect-compensating species, with lowest predicted band gap for FA_mod-25DABA and FA_mod-26DABA (1.2 eV, Figures S54-55, Table S6). We also simulated the electronic structure of UiO-66- $\mathrm{NH}_{2}$ 33\% using the cluster model (Figure S55), obtaining a very similar result to that observed for UiO-66- $\mathrm{NH}_{2}$ when the full periodic structure is taken into account, i.e. the HOMO is localised on the ABDC linker. ${ }^{18-20}$ It is known that the band gap shrinkage in UiO-66$\mathrm{NH}_{2}$ is due to the generation of a new gap state in the proximity of the valence band. ${ }^{18}$ Given the similar situation observed for our defect-engineered MOFs, we infer that the shrinkage of the band gap is likely to be mainly produced by a shift of the valence band to higher energy.

To probe the photocatalytic activity of the materials, the defect-engineered MOFs obtained by PSDE were tested for a challenging gas phase reaction: $\mathrm{CO}_{2}$ photoreduction, one of the two reactions related to solar fuel production. Recent work has shown that indeed MOFs can photoreduce $\mathrm{CO}_{2}$, albeit with low efficiencies so far. ${ }^{28}$ The photocatalytic activity towards reduction of $\mathrm{CO}_{2}$ in the gas phase of FA_mod, FA_mod-2ABA and FA_mod-35DABA was investigated. The setup described in the ESI (Figure S56) was used, depositing the MOFs (30 mg) on a stainless steel metal disc and irradiating for 6 hours with a Xe lamp (300 W) emitting at wavelengths above $325 \mathrm{~nm}$ (Figure S57), under a $1.5: 1 \quad \mathrm{CO}_{2} / \mathrm{H}_{2}$ atmosphere. $\mathrm{P} 25 \mathrm{TiO}_{2}$ was also measured as a reference material in the same conditions. Before the tests, the MOF powders were further washed with water and acetone to remove any trace of DMF from the pores, and therefore any potential source of $\mathrm{CO}$ other than $\mathrm{CO}_{2}$ (Figures S58-60). Figure 4 shows the results of these tests, demonstrating that all materials photoreduced $\mathrm{CO}_{2}$ to $\mathrm{CO}$ with a $100 \%$ selectivity. Both FA_mod-2ABA and FA_mod35-DABA outperform FA_mod. In order to prove that the produced $\mathrm{CO}$ effectively derived from $\mathrm{CO}_{2}$, we performed a control experiment using FA_mod-2ABA as the catalyst and ${ }^{13} \mathrm{CO}_{2}$. ${ }^{13} \mathrm{CO}$ was detected, confirming its origin was from the gaseous stream and not the material and/or traces of solvent (Figure S61). We note that the performance of FA_mod-2ABA is comparable to that of $\mathrm{P} 25$, which is significant, given that recent reports on gas phase $\mathrm{CO}_{2}$ photoreduction using MOFs typically point towards lower performance of the MOFs compared to P25. ${ }^{29}, 30$ Further analyses on charge carrier dynamics should be conducted to explain this improved catalytic activity.

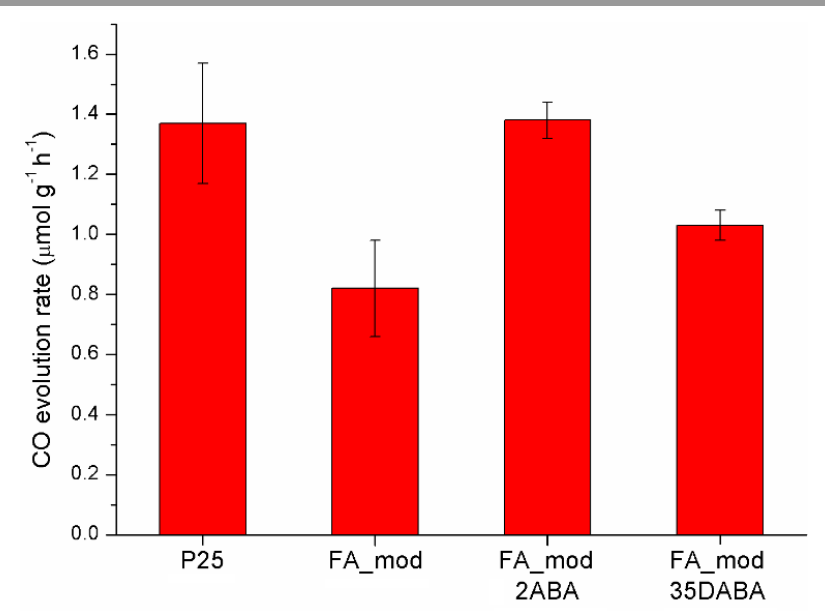

Figure 4. Observed $\mathrm{CO}$ evolution rate after 6 hours of UV vis irradiation with $\mathrm{H}_{2}$ as sacrificial agent, for $\mathrm{TiO}_{2}$ P25, FA_mod, FA_mod-2ABA and FA_mod-35DABA (Xe light source, $300 \mathrm{~W})$.

The ability of the defect-engineered MOFs obtained by PSDE to function as photocatalysts was also evaluated for degradation of the dye Rhodamine $B$ in water. The tests were performed by suspending $20 \mathrm{mg}$ of each MOF in $20 \mathrm{~mL}$ of a $0.008 \mathrm{mM}$ solution of Rhodamine B in water inside a glass vial. Each MOF was tested both in the dark and under light irradiation (visible and $365 \mathrm{~nm}$ UV light), keeping the suspension under vigorous stirring (see ESI for additional details). This was done to isolate the contribution of adsorption of the dye within the porous structure of the MOFs from that of photocatalytic degradation. The absorbance at $554 \mathrm{~nm}$ was taken as indicative of the concentration of Rhodamine B in solution (Figures S62-64). Figure 5 shows that FA_mod behaves exactly the same in the dark and under irradiation, suggesting that disappearance of the dye is purely due to adsorption. On the other hand, FA_mod-35DABA is able to adsorb a smaller fraction of dye than FA_mod, but its efficiency improves when irradiated, suggesting that the dye is being photocatalytically degraded (Figure 5). Similar results were obtained with the other defectengineered MOFs (Figures S65-70). NMR analysis of the recovered powders after the tests suggests that part of the defect-compensating groups was lost during the experiment, most likely replaced by water/hydroxide couples (Figures S7174). The MOFs tested under light irradiation systematically lost more ABAs, which is likely to be the result of combined effect of displacement by water and prolonged exposure to light. This leaching of ABAs from the defect/engineered MOFs could play 
a role in the observed enhancement in dye removal, either by improved adsorption, due to increased porosity of the MOF, or by increased photocatalytic activity of the free ABA released in solution. Therefore, we ran two control experiments: one where 15 mg of FA_mod and 5 mg of 35DABA were separately added to the rhodamine $B$ solution, another one where only 5 mg of 35DABA was employed (Figures S75-78). These two tests saw very little removal of dye, suggesting that the improved performance observed when using FA_mod-35DABA is in fact due to photocatalytic degradation promoted by the defectengineered MOF.
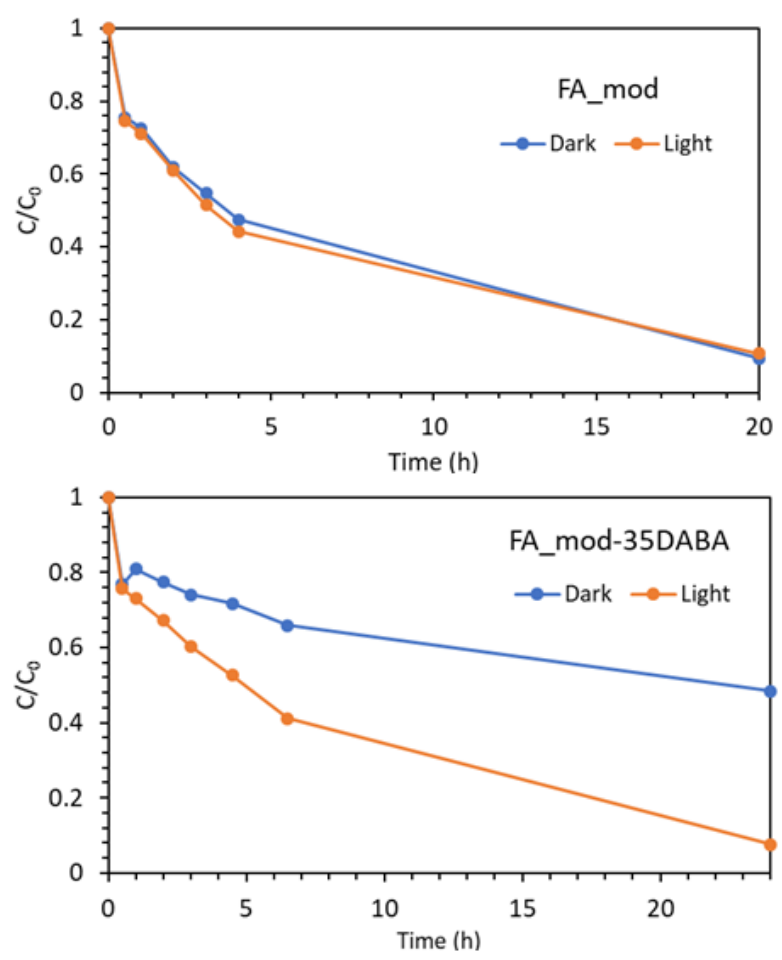

Figure 5. Evolution of the concentration of Rhodamine $B$ in solution (expressed as $C / C_{0}$ $C_{0}=0.008 \mathrm{mM}$ ) during experiments carried out in the dark (blue) and under light irradiation (orange) using $1 \mathrm{mg} / \mathrm{mL}$ of FA_mod (top) and FA_mod-35DABA (bottom).

\section{Conclusions}

We have provided proof of concept that the band gap of the prototypical Zr-MOF UiO-66 can be modulated through a defect-engineering approach, introducing commercially available amino-functionalised monocarboxylates as defectcompensating species, both post-synthesis and by modulated synthesis. The shrinking of the band gap in defect-engineered MOFs mainly arises from the generation of a new HOMO, localised on the grafted aminobenzoic acids, as shown by DFT calculations. The defect-engineered MOFs display improved photocatalytic properties, compared to the pristine defective MOF containing formic acid as defect-capping species. Notably, FA_mod-2ABA displays catalytic activity towards selective $\mathrm{CO}_{2}$ reduction to $\mathrm{CO}$ comparable to that of $\mathrm{TiO}_{2} \mathrm{P} 25$. The main advantage of the defect-engineering approach is that it allows a larger number of degrees of freedom, in terms of position and number of amine groups, than the classical method involving linker modification. We anticipate that the defect-engineering approach could be readily applied to introduce other functional groups able to absorb light and to systematically tune the photochemical properties of the framework, thus contributing to further expand the toolbox available to chemists to modify the physical-chemical properties of MOFs.

\section{Conflicts of interest}

There are no conflicts to declare.

\section{Acknowledgements}

The authors acknowledge the European Union's Horizon 2020 research and innovation programme under the Marie Skłodowska-Curie grant agreement No 663830 (M.T. and M.E.A.W.), and the Engineering and Physical Sciences Research Council (EPSRC) for funding through the First Grant scheme EP/R01910X/1 (M.T. and M.J.M.) and through the CDT in Advanced Characterization of Materials EP/L015277/1 (G.M.S. and C.P.). This work used the Cirrus UK National Tier-2 HPC Service at EPCC (http://www.cirrus.ac.uk) funded by the University of Edinburgh and EPSRC (EP/P020267/1). We would like to acknowledge the assistance provided by the Swansea University AIM Facility, which was funded in part by the EPSRC $\mathrm{EP} / \mathrm{M028267/1}$, the ERDF through the Welsh Government grant 80708, and the Sêr Solar project via the Welsh Government. Stephen J. I. Shearan (Swansea University) is acknowledged for help in collecting NMR data. Prof Paul M. Williams (Swansea University) is acknowledged for providing access to the gas sorption analyser.

\section{Notes and references}

1. Z. Fang, B. Bueken, D. E. De Vos and R. A. Fischer, Angew. Chem. Int. Ed., 2015, 54, 7234-7254.

2. D. S. Sholl and R. P. Lively, J. Phys. Chem. Lett., 2015, 6, 3437-3444.

3. A. K. Cheetham, T. D. Bennett, F. X. Coudert and A. L. Goodwin, Dalton Trans., 2016, 45, 4113-4126.

4. M. Taddei, Coord. Chem. Rev., 2017, 343, 1-24.

5. G. C. Shearer, S. Chavan, S. Bordiga, S. Svelle, U. Olsbye and K. P. Lillerud, Chem. Mater., 2016, 28, 3749-3761.

6. G. C. Shearer, J. G. Vitillo, S. Bordiga, S. Svelle, U. Olsbye and K. P. Lillerud, Chem. Mater., 2016, 28, 7190-7193.

7. O. V. Gutov, M. Gonzalez Hevia, E. C. Escudero-Adan and A. Shafir, Inorg. Chem., 2015, 54, 8396-8400.

8. W. Liang, C. J. Coghlan, F. Ragon, M. Rubio-Martinez, D. M. D'Alessandro and R. Babarao, Dalton Trans., 2016, 45, 4496-4500.

9. X. Feng, H. S. Jena, K. Leus, G. Wang, J. Ouwehand and P. Van Der Voort, J. Catal., 2018, 365, 36-42.

10. A. Schaate, P. Roy, A. Godt, J. Lippke, F. Waltz, M. Wiebcke and P. Behrens, Chem. Eur. J., 2011, 17, 66436651. 
11.

M. J. Cliffe, W. Wan, X. Zou, P. A. Chater, A. K. Kleppe, M. G. Tucker, H. Wilhelm, N. P. Funnell, F. X. Coudert and A. L. Goodwin, Nat. Comm., 2014, 5, 4176.

12. M. Taddei, R. J. Wakeham, A. Koutsianos, E. Andreoli and A. R. Barron, Angew. Chem. Int. Ed., 2018, 57, 1170611710.

13. A. Koutsianos, E. Kazimierska, A. R. Barron, M. Taddei and E. Andreoli, Dalton Trans., 2019, 48, 3349-3359.

14. R. J. Marshall, C. L. Hobday, C. F. Murphie, S. L. Griffin, C. A. Morrison, S. A. Moggach and R. S. Forgan, J. Mater. Chem. A, 2016, 4, 6955-6963.

15. O. V. Gutov, S. Molina, E. C. Escudero-Adan and A. Shafir, Chem. Eur. J., 2016, 22, 13582-13587.

16. S. M. Chavan, G. C. Shearer, S. Svelle, U. Olsbye, F. Bonino, J. Ethiraj, K. P. Lillerud and S. Bordiga, Inorg. Chem., 2014, 53, 9509-9515.

17. E. Flage-Larsen, A. Røyset, J. H. Cavka and K. Thorshaug, J. Phys. Chem. C, 2013, 117, 20610-20616.

18. K. Hendrickx, D. E. Vanpoucke, K. Leus, K. Lejaeghere, A. Van Yperen-De Deyne, V. Van Speybroeck, P. Van Der Voort and K. Hemelsoet, Inorg. Chem., 2015, 54, 1070110710.

19. M. Taddei, D. Tiana, N. Casati, J. A. van Bokhoven, B. Smit and M. Ranocchiari, Phys. Chem. Chem. Phys., 2017, 19, 1551-1559.

20. T. Musho, J. Li and N. Wu, Phys. Chem. Chem. Phys., 2014, 16, 23646-23653.

21. S. J. Garibay, I. Iordanov, T. Islamoglu, J. B. DeCoste and O. K. Farha, CrystEngComm, 2018, 20, 7066-7070.

22. C. Atzori, G. C. Shearer, L. Maschio, B. Civalleri, F. Bonino, C. Lamberti, S. Svelle, K. P. Lillerud and S. Bordiga, J. Phys. Chem. C, 2017, 121, 9312-9324.

23. J. K. Bristow, K. L. Svane, D. Tiana, J. M. Skelton, J. D. Gale and A. Walsh, J. Phys. Chem. C, 2016, 120, 9276-9281.

24. D. Yang, V. Bernales, T. Islamoglu, O. K. Farha, J. T. Hupp, C. J. Cramer, L. Gagliardi and B. C. Gates, J. Am. Chem. Soc., 2016, 138, 15189-15196.

25. D. Yang, S. O. Odoh, T. C. Wang, O. K. Farha, J. T. Hupp, C. J. Cramer, L. Gagliardi and B. C. Gates, J. Am. Chem. Soc., 2015, 137, 7391-7396.

26. L.-M. Yang, E. Ganz, S. Svelle and M. Tilset, J. Mater. Chem. C, 2014, 2, 7111-7125.

27. C. H. Hendon, D. Tiana, M. Fontecave, C. Sanchez, L. D'Arras, C. Sassoye, L. Rozes, C. Mellot-Draznieks and A. Walsh, J. Am. Chem. Soc., 2013, 135, 10942-10945.

28. I. I. Alkhatib, C. Garlisi, M. Pagliaro, K. Al-Ali and G. Palmisano, Catalysis Today, 2018.

29. A. Crake, K. C. Christoforidis, A. Gregg, B. Moss, A. Kafizas and C. Petit, Small, 2019, 15, 1805473.

30.

\section{TABLE OF CONTENTS}

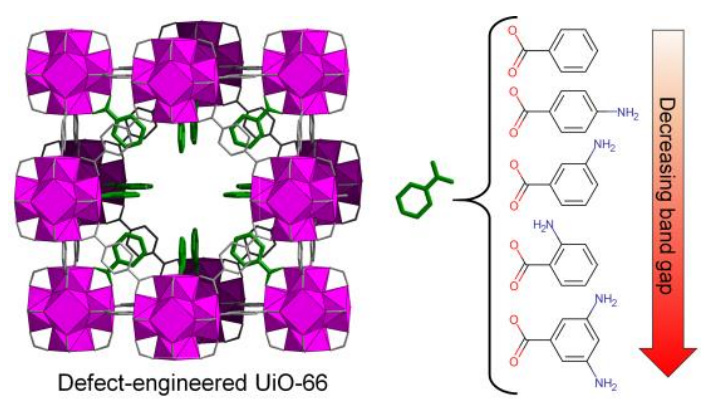

A simple defect engineering approach to systematically tune the band gap of the prototypical zirconium-based metalorganic framework UiO-66 is reported. Defect engineered materials display enhanced photocatalytic activity. 